\title{
A novel approach to motion modeling using fuzzy cognitive map and artificial potential fields
}

\begin{abstract}
Artificial potential field (APF) is well established for reactive navigation of robots. Initially, this paper describes a fast and robust fuzzy-APF on an ActivMedia AmigoBot platform. Obstacle-related information is fuzzified just by sensory fusion which results in shorter runtime. The membership functions of obstacles' range and direction have been also merged into one function for smaller block of rules. The fuzzy-APF is tested and verified virtually with non-concave obstacles. Main contribution of this article is a new approach to motion modeling. The goal is to discover decision making behaviors of the robot in wayfinding. A novel decision modeling technique is developed based on capabilities of the fuzzy cognitive map (FCM) and supervised learning using the genetic algorithm (GA). Decision productions for moving from one sub-space to another are modeled in form of decision matrices. The robot trajectory under supply of such decision matrices has likelihood of nearly $90 \%$ with its trajectory under the APF. Replication of robot motion is therefore achieved by modeling its decision behaviors in form of tangible matrices.
\end{abstract}

Keyword: Potential fields; Fuzzy cognitive map 